\title{
Acaricidal Activity of Ethulia conyzoides Extracts and Constituents against Tetranychus urticae Koch
}

\author{
A. Mahmoud Dawidar \\ Chem. Dept., Fac. of Sci., \\ Mansoura University \\ Mansoura, Egypt
}

\author{
M. El-Hoseiny Mostafa \\ Plant Protection Research \\ Institute, A.R.C., Egypt
}

\author{
M. Abdel-Mogib \\ Chem. Dept., Fac. of Sci., \\ Mansoura University \\ Mansoura, Egypt
}

\author{
M. El Saied. El-Naggar \\ Plant Protection Research \\ Institute, A.R.C., Egypt
}

\begin{abstract}
The acaricidal potential of Ethulia conyzoides L. aerial parts extracts as well as its isolated compounds were investigated against larvae and adults of Tetranychus urticae Koch under laboratory conditions. Chromatographic separation of the extracts gave 3-O-acetyl lupeol, lupan-3-ol, ethuliacoumarin (1) and 7-O-methyl apigenin (Genkwanin), besides a mixture of isoethyliacoumarin A (2), isoethuliacoumarin C (3). The biological evaluation results indicated that ethyl acetate fraction and ethuliacoumarin (1) were the most potent to larvae and adults of Tetranychus urticae. The LC 50 values of ethyl acetate fraction were found to be 11.58 and $17.86 \mathrm{ppm}$, respectively and $\mathrm{LC}_{50}$ values of (1) were found to be 12.72 and $19.22 \mathrm{ppm}$, respectively after 7 days of treatment.
\end{abstract}

Keywords: Acaricidal activity, Tetranychus urticae Koch, Ethulia conyzoides, 3-O-acetyl lupane, lupan-3-ol, ethuliacoumarin, 7-O-methyl apigenin, isoethyliacoumarin A and isoethuliacoumarin C.

\section{INTRODUCTION}

The two spotted spider mite, Tetranychus urticae Koch (Acari: Tetranychidae), is a worldwide damaging pest attacking cotton, fruit trees and vegetables in Egypt [1]. Synthetic chemical acaricides has been the major tool in pest control operations. However, the extensive and repeated use of synthetic organic pesticides have led to environmental problems to the human health, non-target organisms and cause some disastrous ecological damage [2].

The move towards green pesticides represents a new trend for discovering natural pesticides. Natural products considered to be an excellent alternative to synthetic pesticides as a mean to reduce negative impacts to human health and the environment [3].

Ethulia conyzoides L., a wild growing Egyptian plant, has for centuries been used in folk medicine as an anti-helminthic for round worms and for abdominal disorders [4, 5], a source of natural antioxidants [6] and antihypertensive agents. It was used to cure headaches and dysmenorrhea [7]. In addition, the methanol extract of the aerial parts of E. conyzoides has antibacterial activity [5].

The molluscicidal activity of E. conyzoides was recorded [4], which was attributed to the presence of ethuliacoumarin $\mathrm{A}$ and isoethuliacoumarin $\mathrm{A}$.

The previous phytochemical screening revealed that E. conyzoides contains terpenoid 5-methylcoumarins $[5,8,9,10,11,12]$, triterpenoids [13, 14], flavonoids [15] and sterols [13].

This article presents the results of phytochemical and acaricidal activity investigation of $E$. conyzoides.

\section{MATERIALS AND METHODS}

\subsection{General}

NMR experiments were performed on a Bruker AMX 400 instrument standard pulse sequences operating at $400 \mathrm{MHz}$ in ${ }^{1} \mathrm{H}-\mathrm{NMR}$ and ${ }^{13} \mathrm{C}-\mathrm{NMR}$. Chemical shifts are given in $\delta(\mathrm{ppm})$ relative to TMS as internal standard material and the coupling constants $(J)$ are in $\mathrm{Hz}$.

GC/MS analysis of the volatile fractions were performed on a Varian GC interfaced to Finnegan SSQ 7000 Mass selective Detector (SMD) with ICIS V2.0 data system for MS identification of the GC components, at Agriculture Research Center, Dokki, Cairo. The column used was DB-5 (J\&W Scientific, Folosm, CA) cross-linked fused silica capillary column (30 m. long, $0.25 \mathrm{~mm}$. internal diameter) coated with poloy dimethyl-siloxane $(0.5 \mu \mathrm{m}$. film thickness). The oven temperature was programmed from $50^{\circ} \mathrm{C}$ for $3 \mathrm{~min}$., at isothermal, then heating by $7^{\circ} \mathrm{C} / \mathrm{min}$. to $250^{\circ} \mathrm{C}$ and isothermally for $10 \mathrm{~min}$., at $250^{\circ} \mathrm{C}$. Injector temperature was $200^{\circ} \mathrm{C}$ and the volume injected was $0.5 \mu \mathrm{l}$. Transition-line and ion source temperatures were $250^{\circ} \mathrm{C}$ and $150^{\circ} \mathrm{C}$, respectively. The mass spectrometer had a delay of 3 min. to avoid the solvent plead and then scanned from $\mathrm{m} / \mathrm{z} 50$ to $\mathrm{m} / \mathrm{z} 300$. Ionization energy was set at 70 $\mathrm{eV}$.

\subsection{Chemicals}

Normal CC: column chromatography was performed on silica gel Merck grain size 0.2-0.063 mm; preparative TLC were performed on silica gel 
(Kieselgel 60, F 254) of $0.25 \mathrm{~mm}$ thickness. Pet. ether $\left(60-80^{\circ} \mathrm{C}\right)$, methylene chloride, ethyl acetate and methanol were obtained from Adwic Company.

\subsection{Plant material}

The aerial parts of Ethulia conyzoides L. was collected on canal banks near Mansoura in April, 2009, identified by Prof. Dr. Loutfy Boulos, Professor of Botany, Faculty of Science, Alexandria University, Egypt.

\subsection{Extraction and isolation}

The aerial parts of E. conyzoides $(1 \mathrm{Kg})$ were extracted by soxhlet apparatus using different organic solvents of different polarities composed of pet. ethermethylene chloride- ethyl acetate and methanol successively. In order to obtain four fractions, pet. ether fraction $(20.40 \mathrm{~g})$, methylene chloride fraction $(9.28 \mathrm{~g})$, ethyl acetate fraction $(3.05 \mathrm{~g})$ and methanol fraction $(53.72 \mathrm{~g})$, which was further extracted by butanol to give the butanol fraction $(2.31 \mathrm{~g})$.

E. conyzoides fresh aerial parts $(250 \mathrm{~g})$ were processed in order to obtain the volatile oil fraction by means of hydro-distillation technique $(0.54 \mathrm{~g}, 0.22 \%$ fresh weight).

\subsection{Separation of compounds}

The pet. ether fraction $(9.45 \mathrm{~g})$ was defatted using cold methanol to give the defatted pet. ether fraction $(9.42$ g). The defatted pet ether residue was subjected to column chromatography using silica gel as adsorbent. Elution of the column was performed by using a series of eluents from hexane/ acetone combinations of increasing polarity. The effluents were combined into four sub-fractions according to their TLC patterns.

Sub-fraction 1 a yellow orange oily material which was eluted by hexane/ acetone (4: 1) was further purified using preparative TLC eluted by (hexane / ether 3:1) to yield white needle crystals, $R_{f}=0.69$ of 3-O-acetyl lupeol.

Pet. ether fraction afforded by GC/MS technique 6 compounds as indicated by table 1 .

Methylene chloride fraction $(9.28 \mathrm{~g})$ was chromatographed over silica gel CC using a series of eluents from pet. ether / ethyl acetate combinations of increasing polarities. The effluents were recombined into eight sub-fractions based on their TLC patterns.

The sub-fraction 2 eluted by pet. ether / ethyl acetate (13:7) gave by preparative TLC (silica gel, benzene / ether $4: 1$ ) lupan-3-ol at $R_{f}=0.28$ and compound (1) at $R_{f}=0.53$.

The sub-fraction 3 was purified using preparative TLC (silica gel, $\mathrm{CHCl}_{3} / \mathrm{MeOH}(39: 1)$ ) to afford a mixture of compounds (2) and (3) at $R_{f}=0.19$.

Methylene chloride fraction afforded by GC/MS technique 5 compounds as indicated by table 1 .

The ethyl acetate fraction (3.05 g) was purified on sephadex-LH-20, which was washed with $\mathrm{MeOH}$, the effluents were combined into five sub fractions based on their TLC patterns.

Sub-fraction 5 was further purified using preparative TLC developed in a mixture of benzene / acetone
(3:2), where 7-O-methyl apigenin was obtained at $R_{f}$ $=0.67$.

The volatile oil fraction $(0.54 \mathrm{~g}, 0.22 \%)$ was separated by GC/MS to afford 8 compounds.

\subsection{Maintance of spider mite colony}

Colony of spider mite Tetranychus urticae Koch was reared under laboratory condition $\left(25 \pm 2{ }^{\circ} \mathrm{C}\right.$ and $60 \pm 5$ $\%$ R.H) at plant protection research institute branch, Dakahlia Governorate. This study colony was isolated from heavily infested castor oil plant leaves. Spider mite colony was reared on castor oil leaves. These leaves were cleaned and placed on moisten cotton wool pad in Petri dishes. This colony was left for one year under the precious conditions in order to get a homogenous and sensitive colony. Spider mite individual were transferred to the leaves by the aid of fine camels hair brush. Breeding leaves were changed twice weekly at summer and once weekly at winter. Adding water was done twice daily to prevent escaping of $T$. urticae individuals.

\subsection{Assessment of acaricidal activity}

In this respect, laboratory experiments are conducted to evaluate the activity of various tested plant extracts against $T$. urticae mobile stages (larvae and adult females). The leaf-dip technique was used Gazal et al., (1992) [16].

The indication of mortality was chosen as the failure of mites to respond positively by leg movement followed light brooding with a fine brush. Mortality percentages were determined and corrected by using Abotts, (1925) formula [17] and they are statistically analyzed to estimate $\mathrm{LC}_{50}, \mathrm{LC}_{90}$ and slope values according to Finney, (1971) [18]. Toxicity index was computed for different extracts and their isolated compounds by comparing these materials with the most effective extracts or isolated compounds using Sun's, (1950) equation [19].

$$
\text { Toxicity index }=\frac{L C_{50} \text { of compound } A}{L C_{50} \text { of compound } B} \times 100
$$

Where:

A is the most effective compound

$\mathrm{B}$ is the tested compound

\section{RESULTS AND DISCUSSION}

The chromatographic separation of the plant extracts and the spectral analysis of the separated compounds revealed the identification of the triterpenoids lupyl acetate [20] and was previously isolated from the same plant species [13] and lupeol [21]) that was isolated before from E. conyzoides [13], The monoterperpenoidal 5-methylcoumarins ethuliacoumarin (1) which was isolated previously from the same plant species by Baldaa et al., (1980) and Mahmoud et al., (1998) [8, 9], isoethyliacoumarin A (2) and isoethuliacoumarin C (3) which were isolated previously from the same plant species by (Baldaa et al., 1980) [8], as well as the flavonoid 7-O-methyl apigenin (Genkwanin) [22], which has not been isolated previously from $E$. conyzoides.

The pet ether, methylene chloride and the volatile oil fractions of the aerial parts of E. conyzoides were 
analyzed by GC/MS technique. Nineteen compounds which are listed in table 1 were identified.

Table 1: Chemical constituents identified in fractions of $E$. conyzoides aerial parts by GC/MS technique.

\begin{tabular}{|c|c|c|c|c|}
\hline Component name & $\begin{array}{c}\mathbf{R}_{\mathbf{t}} \\
\mathbf{m i n}\end{array}$ & $\begin{array}{c}\text { Area } \\
\%\end{array}$ & M.F & (Identity) $\mathbf{m} / \mathbf{z}$ (ret. int. \%) \\
\hline \multicolumn{5}{|l|}{ Pet. ether fraction } \\
\hline $\begin{array}{c}\text { 4,4-dimethyl- } \\
\text { Tetracyclo[6.3.2.0 } \\
(2,5) .0(1,8)] \text { tridecan-9- } \\
\text { ol (4) }\end{array}$ & 15.17 & 0.80 & $\mathrm{C}_{15} \mathrm{H}_{24} \mathrm{O}$ & $\begin{array}{l}220(1)\left[\mathrm{M}^{+}\right], 205(3)\left[\mathrm{M}-\mathrm{CH}_{3}\right]^{+}, 187(7)\left[\mathrm{M}-\mathrm{CH}_{3}-\mathrm{H}_{2} \mathrm{O}\right]^{+}, 163 \\
(10)\left[\mathrm{C}_{11} \mathrm{H}_{15} \mathrm{O}\right]^{+}, 136(100)\left[\mathrm{C}_{9} \mathrm{H}_{12} \mathrm{O}\right]^{++}, 91(37)\left[\mathrm{C}_{7} \mathrm{H} 7\right]^{+}, 65 \\
(20)\left[\mathrm{C}_{5} \mathrm{H}_{5}\right]^{+}\end{array}$ \\
\hline $\begin{array}{l}\text { Isoaromadendrene } \\
\text { epoxide (5) }\end{array}$ & 15.63 & 0.43 & $\mathrm{C}_{15} \mathrm{H}_{24} \mathrm{O}$ & $\begin{array}{l}220(7)\left[\mathrm{M}^{+}\right], 205(13)\left[\mathrm{C}_{15} \mathrm{H}_{25}\right]^{+}, 149(43)\left[\mathrm{C}_{11} \mathrm{H}_{17}\right]^{+}, 134(96) \\
{\left[\mathrm{C}_{10} \mathrm{H}_{14}\right]^{+}, 105(98)\left[\mathrm{C}_{8} \mathrm{H}_{9}\right]+{ }^{+}, 91(100)\left[\mathrm{C}_{7} \mathrm{H}_{7}\right]^{+}, 77(67)} \\
{\left[\mathrm{C}_{6} \mathrm{H}_{5}\right]^{+}, 65(27)\left[\mathrm{C}_{5} \mathrm{H}_{5}\right]^{+}}\end{array}$ \\
\hline $\begin{array}{l}\text { Perhydrofarnesyl } \\
\text { acetone }\end{array}$ & 17.80 & 1.17 & $\mathrm{C}_{18} \mathrm{H}_{36} \mathrm{O}$ & $\begin{array}{l}268(1)\left[\mathrm{M}^{+}\right], 250(13)\left[\mathrm{C}_{18} \mathrm{H}_{34}\right]^{+}, 225(1)\left[\mathrm{C}_{16} \mathrm{H}_{33}\right]^{+}, 109(35) \\
{\left[\mathrm{C}_{8} \mathrm{H}_{13}\right]^{+}, 85} \\
{\left[\mathrm{C}_{3} \mathrm{H}_{6} \mathrm{O}\right]^{+}}\end{array}$ \\
\hline $\begin{array}{l}\text { 2,3,5-trimethyl-7H- } \\
\text { Furo[3,2- } \\
\text { g][1]benzopyran-7-one }\end{array}$ & 20.76 & 2.53 & $\mathrm{C}_{14} \mathrm{H}_{12} \mathrm{O}_{3}$ & $\begin{array}{l}228(1)\left[\mathrm{M}^{+}\right], 227(3)[\mathrm{M}-\mathrm{H}]^{+}, 213(7)\left[\mathrm{M}-\mathrm{CH}_{3}\right]^{+}, 199(10) \\
{[\mathrm{M}-\mathrm{H}-\mathrm{CO}]^{+}, 185(100)\left[\mathrm{M}-\mathrm{CH}_{3}-\mathrm{CO}^{+}, 175(100)\left[\mathrm{M}-\mathrm{CH}_{3}-\right.\right.} \\
\mathrm{CO}-\mathrm{CO}]^{+}, 91(37)\left[\mathrm{C}_{7} \mathrm{H}_{7}\right]^{+}, 77(67)\left[\mathrm{C}_{6} \mathrm{H}_{5}\right]^{+}\end{array}$ \\
\hline Phytol isomer & 21.08 & 1.50 & $\mathrm{C}_{20} \mathrm{H}_{40} \mathrm{O}$ & $\begin{array}{l}297(1)\left[\mathrm{M}^{+}\right], 123(74)\left[\mathrm{C}_{9} \mathrm{H}_{15}\right]^{+}, 95(78)\left[\mathrm{C}_{7} \mathrm{H}_{11}\right]^{+}, 71(100) \\
{\left[\mathrm{C}_{5} \mathrm{H}_{11}\right]^{+}, 55(63)\left[\mathrm{C}_{4} \mathrm{H}_{7}\right]^{+} .}\end{array}$ \\
\hline Stigmasterol & 35.58 & 3.74 & $\mathrm{C}_{29} \mathrm{H}_{48} \mathrm{O}$ & $\begin{array}{l}412(100)\left[\mathrm{M}^{+}\right], 394(13)\left[\mathrm{M}-\mathrm{H}_{2} \mathrm{O}\right]^{+}, 300(43)\left[\mathrm{C}_{21} \mathrm{H}_{32} \mathrm{O}\right]^{+}, 255 \\
(54)\left[\mathrm{C}_{19} \mathrm{H}_{27}\right]^{+}, 231(12)\left[\mathrm{C}_{16} \mathrm{H}_{23} \mathrm{O}\right]^{+}, 213(30)\left[\mathrm{C}_{16} \mathrm{H}_{21}\right]^{+}, 133 \\
(43)\left[\mathrm{C}_{10} \mathrm{H}_{13}\right]^{+}, 55(70)\left[\mathrm{C}_{4} \mathrm{H}_{7}\right]^{+}\end{array}$ \\
\hline \multicolumn{5}{|c|}{ Methylene chloride fraction } \\
\hline $\begin{array}{l}\text { Benzaldehyde,4- } \\
\text { methoxy }\end{array}$ & 12.33 & 1.90 & $\mathrm{C}_{8} \mathrm{H}_{8} \mathrm{O}_{2}$ & $\begin{array}{l}136(100)\left[\mathrm{M}^{+}\right], 135(100)\left[\mathrm{M}^{+}-\mathrm{H}\right]^{+}, 107(13)\left[\mathrm{M}^{+}-\mathrm{CO}\right]^{+}, 105 \\
(7)\left[\mathrm{M}^{+}-\mathrm{OCH}_{3}\right]^{+}, 77(20)\left[\mathrm{C}_{6} \mathrm{H}_{5}\right]^{+}\end{array}$ \\
\hline Coniferyl alcohol & 16.56 & 0.54 & $\mathrm{C}_{10} \mathrm{H}_{12} \mathrm{O}_{3}$ & $\begin{array}{l}180(74)\left[\mathrm{M}^{+}\right], 164(100)\left[\mathrm{M}^{+}-\mathrm{OCH}_{3}\right]^{+}, 137(100)\left[\mathrm{C}_{8} \mathrm{H}_{9} \mathrm{O}_{2}\right]^{+} \\
124(53)\left[\mathrm{C}_{7} \mathrm{H}_{8} \mathrm{O}_{2}\right]^{++}, 91(32)\left[\mathrm{C}_{7} \mathrm{H}_{7}\right]^{+}, 77(20)\left[\mathrm{C}_{6} \mathrm{H}_{5}\right]^{+}\end{array}$ \\
\hline (-)-Loliolide & 17.04 & 1.45 & $\mathrm{C}_{11} \mathrm{H}_{16} \mathrm{O}_{3}$ & $\begin{array}{l}196(26)\left[\mathrm{M}^{+}\right], 178(9)\left[\mathrm{M}^{+}-\mathrm{H}_{2} \mathrm{O}^{+}, 163(13)\left[\mathrm{M}^{+}-\mathrm{H}_{2} \mathrm{O}-\mathrm{CH}_{3}\right]^{+}\right. \\
135(48)\left[\mathrm{C}_{9} \mathrm{H}_{11}\right]^{+}, 111(100)\left[\mathrm{C}_{6} \mathrm{H}_{7} \mathrm{O}_{2}\right]^{+}, 91(78)\left[\mathrm{C}_{7} \mathrm{H}_{7}\right]^{+}, 65 \\
(9)\left[\mathrm{C}_{5} \mathrm{H}_{9}\right]^{+} \text {. }\end{array}$ \\
\hline Neophytadiene & 18.02 & 0.61 & $\mathrm{C}_{20} \mathrm{H}_{38}$ & $\begin{array}{l}278(9)\left[\mathrm{M}^{+}\right], 123(65)\left[\mathrm{C}_{9} \mathrm{H}_{15}\right]^{+}, 95(88)\left[\mathrm{C}_{17} \mathrm{H}_{11}\right]^{+}, 82(77) \\
{\left[\mathrm{C}_{6} \mathrm{H}_{10}\right]^{+}, 71(4)\left[\mathrm{C}_{5} \mathrm{H}_{11}\right]^{+}, 68(100)\left[\mathrm{C}_{5} \mathrm{H}_{8}\right]^{+}, 55(84)\left[\mathrm{C}_{4} \mathrm{H}_{7}\right]^{+}}\end{array}$ \\
\hline Phytol & 21.05 & 0.36 & $\mathrm{C}_{20} \mathrm{H}_{40} \mathrm{O}$ & $\begin{array}{l}297(4)\left[\mathrm{M}^{+}\right], 123(28)\left[\mathrm{C}_{9} \mathrm{H}_{15}\right]^{+}, 95(13)\left[\mathrm{C}_{7} \mathrm{H}_{11}\right]^{+}, 71(100) \\
{\left[\mathrm{C}_{5} \mathrm{H}_{11}\right]^{+}, 55(27)\left[\mathrm{C}_{4} \mathrm{H}_{7}\right]^{+} .}\end{array}$ \\
\hline \multicolumn{5}{|l|}{ volatile oil fraction } \\
\hline Trans-caryophyllene & 11.89 & 0.43 & $\mathrm{C}_{15} \mathrm{H}_{24}$ & $\begin{array}{l}204(13)\left[\mathrm{M}^{+}\right], 189(33)\left[\mathrm{M}_{-} \mathrm{CH}_{3}\right]^{+}, 147(40)\left[\mathrm{C}_{11} \mathrm{H}_{15}\right]^{+}, 133 \\
(100)\left[\mathrm{C}_{10} \mathrm{H}_{13}\right]^{+}, 91(86)\left[\mathrm{C}_{7} \mathrm{H}_{7}\right]^{+}, 55(40)\left[\mathrm{C}_{4} \mathrm{H}_{7}\right]^{+},\end{array}$ \\
\hline $\begin{array}{l}\text { (-)-Caryophyllene } \\
\text { oxide }\end{array}$ & 14.43 & 1.30 & $\mathrm{C}_{15} \mathrm{H}_{24} \mathrm{O}$ & $\begin{array}{l}220(1)\left[\mathrm{M}^{+}\right], 205(7)\left[\mathrm{M}-\mathrm{CH}_{3}\right]^{+}, 149(17)\left[\mathrm{C}_{11} \mathrm{H}_{17}\right]^{+}, 123(17) \\
{\left[\mathrm{C}_{9} \mathrm{H}_{15}\right]^{+}, 109(50)\left[\mathrm{C}_{7} \mathrm{H}_{9} \mathrm{O}\right]^{+}, 93(86)\left[\mathrm{C}_{7} \mathrm{H}_{9}\right]^{+}, 55(40)\left[\mathrm{C}_{4} \mathrm{H}_{7}\right]^{+} .}\end{array}$ \\
\hline $\begin{array}{c}\text { Caryophylla- } \\
4(12), 8(13) \text {-dien-5 } \beta \text {-ol }\end{array}$ & 15.17 & 0.47 & $\mathrm{C}_{15} \mathrm{H}_{24} \mathrm{O}$ & $\begin{array}{l}220(1)\left[\mathrm{M}^{+}\right], 205(2)\left[\mathrm{M}-\mathrm{CH}_{3}\right]^{+}, 187(3)\left[\mathrm{M}-\mathrm{CH}_{3}-\mathrm{H}_{2} \mathrm{O}\right]^{+}, 136 \\
(100)\left[\mathrm{C}_{10} \mathrm{H}_{16}\right]^{+}, 109(20)\left[\mathrm{C}_{7} \mathrm{H}_{9} \mathrm{O}\right]^{+}, 91(34)\left[\mathrm{C}_{7} \mathrm{H}_{7}\right]^{+} .\end{array}$ \\
\hline Hexestrol (6) & 20.55 & 0.20 & $\mathrm{C}_{18} \mathrm{H}_{22} \mathrm{O}_{2}$ & $\begin{array}{l}270(22)\left[\mathrm{M}^{+}\right], 135(100)\left[\mathrm{C}_{9} \mathrm{H}_{11} \mathrm{O}\right]^{+}, 120(7)\left[\mathrm{C}_{8} \mathrm{H}_{8} \mathrm{O}\right]^{+}, 107(3) \\
{\left[\mathrm{C}_{7} \mathrm{H}_{7} \mathrm{O}\right]^{+}, 77(15)\left[\mathrm{C}_{6} \mathrm{H}_{5}\right]^{+} .}\end{array}$ \\
\hline $\begin{array}{l}\text { Acetyl tri-n-butyl } \\
\text { citrate }\end{array}$ & 22.73 & 0.46 & $\mathrm{C}_{20} \mathrm{H}_{34} \mathrm{O}_{8}$ & 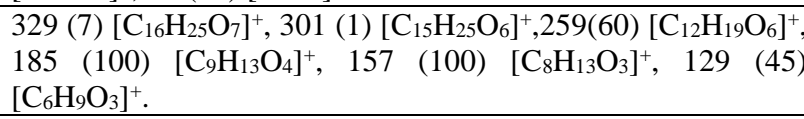 \\
\hline Taraxasterol & 25.28 & 1.36 & $\mathrm{C}_{30} \mathrm{H}_{50} \mathrm{O}$ & $\begin{array}{l}426(53)\left[\mathrm{M}^{+}\right], 411(27)\left[\mathrm{M}-\mathrm{CH}_{3}\right]^{+}, 393(7)\left[\mathrm{M}-\mathrm{CH}_{3}-\mathrm{H}_{2} \mathrm{O}\right]^{+} \\
272(8)\left[\mathrm{C}_{20} \mathrm{H}_{32}\right]^{+}, 207(78)\left[\mathrm{C}_{14} \mathrm{H}_{23} \mathrm{O}\right]^{+}, 135(97)\left[\mathrm{C}_{10} \mathrm{H}_{15}\right]^{+}, \\
95(72)\left[\mathrm{C}_{7} \mathrm{H}_{11}\right]^{+}, 77(17)\left[\mathrm{C}_{6} \mathrm{H}_{5}\right]^{+}, 55(50)\left[\mathrm{C}_{4} \mathrm{H}_{7}\right]^{+} .\end{array}$ \\
\hline$\beta$-Amyrin acetate & 27.52 & 1.52 & $\mathrm{C}_{32} \mathrm{H}_{52} \mathrm{O}_{2}$ & $\begin{array}{l}468(99)\left[\mathrm{M}^{+}\right], 393(49)\left[\mathrm{M}-\mathrm{CH}_{3}-\mathrm{H}_{2} \mathrm{O}\right]^{+}, 218(48)\left[\mathrm{C}_{16} \mathrm{H}_{26}\right]^{+}, \\
203(55)\left[\mathrm{C}_{15} \mathrm{H}_{23}\right]^{+}, 189(81)\left[\mathrm{C}_{14} \mathrm{H}_{21}\right]^{+} .\end{array}$ \\
\hline Lupeol actate & 29.29 & 2.69 & $\mathrm{C}_{32} \mathrm{H}_{52} \mathrm{O}_{2}$ & $\begin{array}{l}68(37)\left[\mathrm{M}^{+}\right], 453(17)\left[\mathrm{M}-\mathrm{CH}_{3}\right]^{+}, 393(10)\left[\mathrm{M}-\mathrm{CH}_{3}\right. \\
\left.\mathrm{CH}_{3} \mathrm{COOH}\right]^{+}, 203(37)\left[\mathrm{C}_{15} \mathrm{H}_{23}\right]^{+}, 189(100)\left[\mathrm{C}_{14} \mathrm{H}_{21}\right]^{+}, 161 \\
(28)\left[\mathrm{C}_{12} \mathrm{H}_{17}\right]^{+}, 135(47)\left[\mathrm{C}_{10} \mathrm{H}_{15}\right]^{+}, 121(47)\left[\mathrm{C}_{9} \mathrm{H}_{13}\right]^{+} .\end{array}$ \\
\hline
\end{tabular}




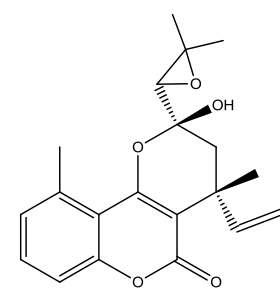

1<smiles>CC1(C)CC2C1C13CCC(O)C1(C)C2CC3</smiles>

4

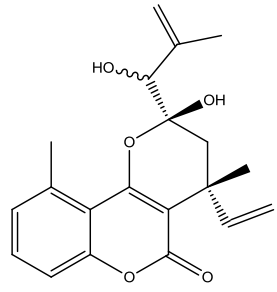

2

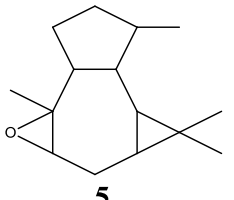<smiles>C=C[C@]1(C)C=C(C(=O)C(C)(C)O)Oc2c1c(=O)oc1cccc(C)c21</smiles>

3

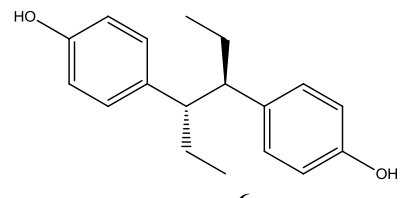

The results of acaricidal activity were presented obtained in table (2) that showed the efficiency of $E$. conyzoides fractions and their isolated compounds against the larvae of $T$. urticae after 7 days of treatment. The ethyl actate fraction was the most effective at $\mathrm{LC}_{50}$ level followed by butanol fraction, pet. ether fraction, methylene chloride fraction and essential oil extract. The $\mathrm{LC}_{50}$ values of these fractions were 11.58, 21.00, 53.63, 76.47 and 282.23 ppm, respectively. Taking the toxicity index into consideration, it could be also observed that ethyl actate fraction was the most effective fraction against the larvae of $T$. urticae after 7-days of treatment followed by butanol fraction, pet. ether fraction, methylene chloride fraction and essential oil extract.

The susceptibility of the adult females of $T$. urticae to various plant fractions after 7-days of treatments (table 2) showed that the LC50 values were 17.86, 53.37, 109.73, 175.94 and $334.73 \mathrm{ppm}$ for ethyl acetate fraction, butanol fraction, methylene chloride fraction, pet. ether fraction and essential oil fraction, respectively.
The toxicity index of the $\mathrm{LC}_{50}$ values showed that ethyl acetate fraction was the most effective plant fraction against adult females of $T$. urticae after 7days of treatment, followed by butanol fraction, methylene chloride fraction and pet. ether fraction. Essential oil fraction was the least effect one.

Bioassay-guided fractionation led to the isolation and purification of six compounds which were investigated as acaricides. The toxicity of the isolated compounds of E. conyzoides fractions against adult females of $T$. urticae were presented in table (2). Ethuliacoumarin (1) was the most effective isolated compound against larvae and adult females of $T$. urticae after 7- days of treatment followed by (isoethyliacoumarin A (2), isoethuliacoumarin C (3)), lupan-3-ol, 3-O-acetyl lupeol and genkwanin.

Our results were in agreement with the finding by Kady et al., (1992) who reported that the molluscicidal principles of $E$. conyzoides were identified as ethuliacoumarin A and isoethuliacoumarin A [4]. 
Table 2. Toxicity of plant fractions and isolated compounds against larvae and adult females of T. urticae after 7- days of treatment.

\begin{tabular}{|c|c|c|c|c|c|c|c|c|}
\hline \multirow[t]{2}{*}{ Plant extract } & \multicolumn{4}{|c|}{ Larvae } & \multicolumn{4}{|c|}{ Adult females } \\
\hline & $\begin{array}{c}\mathbf{L C}_{50}(\mathbf{p p m}) \\
\text { and confidence limits } \\
\text { at } 95 \%\end{array}$ & $\begin{array}{c}\mathrm{LC}_{90}(\mathrm{ppm}) \\
\text { and confidence limits } \\
\text { at } 95 \%\end{array}$ & Slope & $\begin{array}{c}\text { Toxicity } \\
\text { index at } \\
\text { LC }_{50} \\
\text { value }\end{array}$ & $\begin{array}{c}\mathrm{LC}_{50}(\mathrm{ppm}) \\
\text { and confidence limits } \\
\text { at } 95 \%\end{array}$ & $\begin{array}{c}\mathbf{L C}_{90}(\mathrm{ppm}) \\
\text { and confidence limits } \\
\text { at } 95 \%\end{array}$ & Slope & $\begin{array}{c}\text { Toxicity } \\
\text { index at } \\
\text { LC }_{50} \text { value }\end{array}$ \\
\hline \multirow[b]{2}{*}{ Pet. ether fraction } & 53.63 & 1225.41 & \multirow{2}{*}{$0.943 \pm 0.210$} & \multirow[b]{2}{*}{21.59} & 175.94 & 2250.52 & \multirow{2}{*}{$1.104 \pm 0.259$} & \multirow[b]{2}{*}{10.15} \\
\hline & $20.35 \quad 93.05$ & $540.97 \quad 7983.04$ & & & $96.40 \quad 273.84$ & $1108.31 \quad 20981.68$ & & \\
\hline \multirow{2}{*}{$\begin{array}{l}\text { Methylene Chloride } \\
\text { fraction }\end{array}$} & 76.47 & 496.28 & \multirow{2}{*}{$1.578 \pm 0.283$} & \multirow{2}{*}{15.14} & 109.73 & 9095.97 & \multirow{2}{*}{$0.668 \pm 0.189$} & \multirow[b]{2}{*}{16.28} \\
\hline & $50.30 \quad 105.49$ & $304.99 \quad 1242.25$ & & & $38.45 \quad 212.01$ & $1999.50 \quad 14463.1 \mathrm{E}+2$ & & \\
\hline \multirow[b]{2}{*}{ Ethyl acetate fraction } & 11.58 & 130.84 & \multirow{2}{*}{$1.217 \pm 0.270$} & \multirow{2}{*}{100.00} & 17.86 & 100.68 & \multirow{2}{*}{$1.707 \pm 0.320$} & \multirow{2}{*}{100.00} \\
\hline & $\begin{array}{ll}5.69 & 17.59\end{array}$ & $68.55 \quad 578.12$ & & & $10.26 \quad 25.16$ & $67.54 \quad 208.27$ & & \\
\hline \multirow[b]{2}{*}{ Butanol fraction } & 21.00 & 800.43 & \multirow{2}{*}{$0.811 \pm 0.254$} & \multirow{2}{*}{55.14} & 53.37 & 442.08 & \multirow{2}{*}{$1.396 \pm 0.276$} & 2246 \\
\hline & $\begin{array}{ll}4.39 & 38.37\end{array}$ & $245.60 \quad 81924.59$ & & & $31.37 \quad 76.69$ & $254.23 \quad 1373.01$ & & 35.40 \\
\hline & 282.23 & 6146.57 & & & 334.73 & 3762.10 & & $5-24+2$ \\
\hline Essential oil fraction & $116.70 \quad 466.06$ & $2315.09 \quad 11439.8 \mathrm{E}+1$ & $0.958 \pm 0.256$ & 4.10 & $129.12 \quad 532.61$ & $1995.07 \quad 18461.55$ & $1.220 \pm 0.298$ & 5.34 \\
\hline 3-O-acetyl lupeol & 201.64 & 910.73 & $1.957 \pm 0.447$ & 6.31 & 348.61 & 3307.25 & $1.312 \pm 0.275$ & 5.51 \\
\hline lupan-3-ol & $\begin{array}{c}100.93 \quad 285.45 \\
137.21\end{array}$ & $\begin{array}{c}628.18 \quad 1991.13 \\
774.15\end{array}$ & & & $\begin{array}{cc}184.21 \quad 516.21 \\
182.19\end{array}$ & $\begin{array}{cc}1848.00 \quad 11578.63 \\
1609.07\end{array}$ & & \\
\hline & $60.66 \quad 201.32$ & $500.10 \quad 2160.80$ & $1.706 \pm 0.416$ & 9.27 & $87.43 \quad 274.70$ & $951.62 \quad 4858.05$ & $1.354 \pm 0.286$ & 10.55 \\
\hline Ethuliacoumarin (1) & 12.72 & $\begin{array}{c}396.98 \\
\end{array}$ & $0.858 \pm 0.254$ & 100.00 & 19.22 & 863.57 & $0.776 \pm 0.249$ & 100.00 \\
\hline Isoethyliacoumarin A & & & & & 37.31 & $200.91 \quad 59855.5 \mathrm{E}+1$ & & \\
\hline$(2)$ & 35.46 & 261.77 & $1.476 \pm 0.388$ & 35.87 & 66.64 & 452.89 & $1.540 \pm 0.279$ & 28.84 \\
\hline $\begin{array}{l}\text { Isoethuliacoumarin C } \\
\text { (3) }\end{array}$ & $8.36 \quad 60.72$ & $171.33 \quad 684.08$ & 1.7010 .000 & & $44.21 \quad 92.41$ & $271.15 \quad 1209.23$ & & \\
\hline Genkwanin & $\begin{array}{c}297.55 \\
6007\end{array}$ & $\begin{array}{c}3714.43 \\
\end{array}$ & $1.169 \pm 0.370$ & 4.27 & $\begin{array}{c}362.79 \\
0502\end{array}$ & $\begin{array}{c}5745.07 \\
5701433\end{array}$ & $1.068 \pm 0.257$ & 5.30 \\
\hline & $69.07 \quad 489.80$ & $1720.12 \quad 459176.86$ & $1.109 \pm 0.070$ & 4.21 & $195.92 \quad 573.89$ & $2378.06 \quad 57914.33$ & $1.000 \pm 0.251$ & 5.50 \\
\hline
\end{tabular}




\section{REFERENCES}

[1] Dawidar, A. M., Abdel-Mogib, M., El-Naggar, M. E., Mostafa, M. E. (2014). Isolation and Characterization of Polygonum equisetiforme Flavonoids and Their Acaricidal Activity against Tetranychus urticae Koch. Research Journal of Pharmaceutical, Biological and Chemical Sciences vol. 5, issue (4), pp 140-148.

[2] Tehri, K., Singh, N. (2015). The role of botanicals as green pesticides in integrated mosquito management - A review. International Journal of Mosquito Research vol. 2, issue (1), pp 18-23.

[3] Koul, O., Walial, S., Dhaliwal, G. S., (2008). Essential Oils as Green Pesticides: Potential and Constraints. Biopestic. Int. vol. 4, issue (1), pp 63-84.

[4] Kady, M. M., Brimer, L., Furu, P., Lemmich, E., Nielsen, M. H., Thiilborg, S. T., Thastrup, O., Christensen, B. S. (1992). The Molluscicidal Activity of Coumarins from Ethulia conyzoides and of Dicumarol. Planta Med. vol. 58, pp 334337.

[5] El-Bassuony, A. A. (2009). Antibacterial Activity of Two New Monoterpene Coumarins from Ethulia Conyzoides. Journal of Pharmacy Research vol. 2, issue (4), pp 582-584.

[6] Aliyu, A. B., Ibrahim, M. A., Ibrahim, H., Musa, A. M., Lawal, A. Y., Oshanimi, J. A., Usman, M., Abdulkadir, I. E., Oyewale, A. O., Amupitan, J. O. (2012). Free radical scavenging and total antioxidant capacity of methanol extract of Ethulia conyzoides growing in Nigeria. Romanian Biotechnological Letters vol. 17, issue (4), pp 7458- 7465.

[7] Noumia, E., Houngue, F., Lontsi, D. (1999). Traditional medicines in primary health care: plants used for the treatment of hypertension in Bafia, Cameroon. Fitoterapia vol. 70, pp 134139.

[8] Baldaa, S. I.; Halim, A. F.; Halaweish, F. T. and Bohlmann, F. (1980). New 5- methylcoumarin Derivatives from Ethulia conyzoides, Phytochemistry, vol. 19, pp 1519-1522.

[9] Mahmoud, A. A., Ahmed, A. A., Ilnuma, M., Tanaka, T. (1998). Further Monoterpene 5methylcoumarins and an Acetophenone Derivative from Ethulia conyzoides. Phytochemistry vol. 48 issue (3), pp 543546.

[10] Shukla, V. S., Dutta, S. C., Baruah, R. N., Sharma, R. P., Thyagarajan, G., Herz, W., Kumar, N., Watanabe, K., Blount, J. F. (1982).
New 5-methylcoumarins from Ethulia conyzoides. Phyrochemistry vol. 21 issue (7), pp 1725-1731.

[11] Bohlmann, F., Balbaa, S., halim, A., Halawelsh, F. A., (1981). Terpene-Coumarin Derivative from Ethulia conyzoides. Phytochemistry vol. 20, pp 177.

[12] Mahmoud, A. A., Ahmed, A. A., linuma, M., Tanaka, T., Muraoka, O. (1994). Structure of a Novel Spiro-monoterpene-coumarin in Ethulia conyzoides. Tetrahedron Letters vol. 35 issue (35), pp 6517-6520.

[13] Mahmoud, Z. F., Sarg, T. M., Amer, M. E., Khafagy, S. M. (1983). Anthelmintic coumarin from Ethulia conyzoides var. gracilis Asch. \& Schweinf. Pharmazie vol. 38 issue (7), pp 486487.

[14] Balbaa, S. I., Halim, A. F., Halaweish, F. T. (1981). Constituents of the Aerial Parts of Ethulia conyzoides. Fitoterapia vol. 52 issue (2), pp 75-76.

[15] Mahmoud, Z. F., Sarg, T. M., Amer, M. E., Khafagy, S. M., Bohlmann, F. (1980). A 5methylcoumarin Glucoside from Ethulia conyzoides. Phytochemistry vol. 19 pp 20292030.

[16] Gazal, S.A., Abuzarqa, M., Mahasneh, A.M. (1992). Phytother. Res. vol. 6, pp 265-269.

[17] Abbott, W.S. (1925). J Econ Entomol. vol. 18, pp 625-627.

[18] Finney, D.J. (1971). Probit Analysis. A Statistical treatment of the sigmoid Response curve. $7^{\text {th }}$ Ed .Cambridge Univ. Press, Cambridge, England.

[19] Sun, Y.P. (1950). J Econ Entomol. vol. 43, pp 45-53.

[20] Jamal, A.K., Yaacob, W.A., Din, L. B. A. (2008). Chemical Study on Phyllanthus reticulatus. Journal of Physical Science vol. 19 issue (2), pp 45-50.

[21] Chuan-Rui, Z., Aldosari, S. A., Vidyasagar, P. S. P. V., Nair, K. M., Nair, M. G., (2013). Antioxidant and Anti-inflammatory Assays Confirm Bioactive Compounds in Ajwa Date Fruit, J. Agric. Food Chem. vol. 61, pp 5834-5840.

[22] Isaev, I. M., Agzamova, M. A., Isaev, M. I. (2011). Genkwanin and iridoid glycosides from Leonurus turkestanicus. Chemistry of Natural Compounds vol. 47, issue (1), pp 132-134. 